\title{
Compliance Level of Textual Therapeutic Usage of Kshirakakoli Containing Formulations with a Serial Ethnomedicinal Survey and Modern System of Medicine
}

\author{
Gunpreet Kaur $^{1}$, Vikas Gupta ${ }^{1}$, Ravinder Sharma ${ }^{2}$, Sanjiv Kumar ${ }^{3}$, RG Singhal ${ }^{4}$, \\ Ranjit Singh ${ }^{4}$, Parveen Bansal ${ }^{1, *}$ \\ ${ }^{1}$ University Centre of Excellence in Research, Baba Farid University of Health Sciences, Faridkot, Punjab, India \\ ${ }^{2}$ University Institute of Pharmaceutical Sciences, Faridkot, Punjab, India \\ ${ }^{2}$ Regional Ayurveda Research Institute, Ranikhet, Uttarakhand, India \\ ${ }^{3}$ Shobhit University, Meerut, Uttar Pradesh, India \\ ${ }^{4}$ Shobhit University, Gangoh, Uttar Pradesh, India \\ Corresponding author* \\ ucer_bfuhs@rediffmail.com,reetjattana21@gmail.com
}

Manuscript received: 19 November, 2020. Revision accepted: 28 June, 2021. Published: 01 July, 2021.

\begin{abstract}
Fritillaria roylei (Kshirakakoli) is a primal plant used in ancient times. But nowadays, due to biotic and abiotic stress the plant has entered in the list of threatened medicinal plant. In ancient texts effective uses of formulations containing kshirakakoli are well mentioned but the information is not written in simple language due to which the therapeutic value of the plant is not well understood by scientific fraternity. So, there is a major need to perform ethno medicinal survey for the formulations containing kshirakakoli and compare their therapeutic uses as mentioned in text with the modern system of medicine. In this study, a field survey was performed in 4 states i.e., Uttar Pradesh, Uttrakhand, Punjab and Himachal Pradesh where the conversation regarding usage of this plant or formulation was done with 24 local medical practitioners, 18 shopkeepers and 4 traditional healers. The information thus obtained were recorded and then compared. Results showed that the usage of kshirakakoli containing formulations was highest in Himachal Pradesh and Uttrakhand. Only few clinical studies have been done on these formulations. The effectiveness of the formulations against remedies alluded by the trado-medical practitioners claimed was found accurate as per ayurvedic textual literature. Hence, the ethno medicinal survey provides a precise guidance to scientists for future research on these kshirakakoli containing formulations that are useful in plethora of disorders.
\end{abstract}

Keywords: Ethnomedicinal survey; Kshirakakoli; Formulations; Ayurveda; Fritillaria roylei.

\section{INTRODUCTION}

Today, plant products, animal products, minerals and metals are used by pharmaceutical industries for production of majority of the Ayurvedic as well as allopathic medicines. On the basis of information obtained from the traditional healers nearly 121 pharmaceutical products have been discovered (Anesini and Perez, 1993; Kshiti, 2012). The knowledge of medicinal plants started fading away with the desertion of Gurukul system of ancient teaching, as written details of most of the medicinal plants are not available (Sharma and Balkrishna, 2005). This also happened with eight plants of Ashtawarga that are considered as very good rasayana with rejuvenating and health promoting properties that strengthens the immune system. Due to high therapeutic properties, these plants are used in various Ayurvedic formulations like taila (medicated oil), Ghritam (medicated clarified butter), churana (powder) and other formulations of traditional medicinal system including chywanprash and other disease preventive tonics (Dhyani et al., 2010). 'Ashtawarga' a significant constituent of a variety of classical Ayurvedic formulations has been assigned a variety of medicinal properties by ancient Materia Medica dealing with Ayurveda and a subject of rigorous botanical research.

Kshirakakoli known as Fritillaria roylei (one out of group of eight Ashtwarga plants) come under the category of threatened species (Saha et al., 2015). Due to limited distribution in their natural habitat, today substitutes of Ashtawarga plants are commonly used in Ayurvedic formulations to meet the market demand. Department of AYUSH, Govt. of India has suggested use of substitutes in formulations in absence of original plants however this option is being exploited by manufacturers rendering such an important and precious plant in ignored condition (Sagar, 2014; Tewari,1991). Hence it becomes important to highlight the therapeutic potentials of this plant in front of scientists so that a justified research shall be carried out on an important but ignored plant. Fritillaria roylei contain various 
active compounds like peimine, peiminine, peimisine, peimiphine, peimidine, peimitidine, propeimin, sterol and these active compounds possess galactogogue, haemostatic, ophthalmic and cytotoxic properties (Chi et al., 1940; Wu, 1944; Chou, 1947; Chatterjee et al., 1976; Jiang et al., 2006). Due to the lack of authentic species in natural habitats, systemized studies/clinical studies have not been carried out on this group of plants. A very few clinical studies related to its therapeutic potentials have been carried out. Moreover, the information available is also highly scattered that is not organized in proper manner due to which it cannot be used by scientist groups working on such important plants. This plant is being used in a number of formulations of high therapeutic value as mentioned in ancient texts however a very limited data with scientific evidences is available in modern literature as well as on internet sources. Ancient texts claim very potent uses of these formulations but as the information is available in regional languages or in Sanskrit, so the real uses of the plant are not well understood by scientific fraternity. Hence it becomes important to know the real status of the mentioned therapeutic potentials as well as practiced potentials by trado-medical practitioners (TMPs) and put it in front of scientists so as to give a thrust to clinical studies on therapeutic effects as well as pharmacological actions of the plant.

\section{MATERIAL AND METHODS}

In India, particularly in the rural and remote areas, there are number of trado-medical practitioners (TMPs) and various kshirakakoli containing ayurvedic formulations are used by these practitioners as a traditional medicine for the treatment of a variety of ailments. So, a folklore survey was taken in 2019 to recognize the therapeutic importance of available kshirakakoli containing formulations mainly in four states of northern India. A field survey was conducted around and in district headquarters and discussion was done with the 24 local medical practitioners, 18 shopkeepers and 4 traditional healers and the information related to these ayurvedic formulations was recorded. The information procured was validated by comparing the information given by at least four TMPs. The medicinal uses of these formulations were recorded from the folklore claims and the standard literature of the Indian systems of medicine. An effort has been made to highlight the traditional use of these formulations so as to enable the scientists to explore these formulations for further research studies.

\section{RESULT AND DISCUSSION}

The folklore survey was done in various districts in Himachal Pradesh (Shimla, Dharamshala, Kangra, Mandi, Kullu and Manali), Punjab (Sangrur, Barnala, Ludhiana, Bhatinda, Patiala, Ferozepur, Faridkot), Uttrakhand (Dehradun, Haridwar, Pauri Garhwal, Rudraprayag) and in Uttar Pradesh (Agra, Meerut, Moradabad, Mathura). It was observed that usages of 20 formulations containing kshirakakoli were maximum in Himachal Pardesh and Uttrakhand followed by Uttar Pradesh and least in Punjab. The excerpts of ayurvedic formulations containing kshirakakoli as one of the ingredients are listed in Table 1 . The compliance level of therapeutic uses of kshirakakoli containing formulations as per ancient literature with trado-medical practitioners (TMPs) and Pre-clinical/clinical trials/case studies as per modern system of medicine are enumerated as in Table 2. These formulations have been indicated in a plethora of reproductive disorders, loss of digestion, insanity, depression, depletion of body tissue, emaciation, phthisis, cures gout arthritis pervading the whole body, heart disease, facial paralysis, diseases of the head/neck and epilepsy. Similar type of ethnomedicinal survey was conducted by the authors for Kakoli containing formulations (Kaur et al., 2019). So, there is a need of hour to explore Kakoli and Kshirakakoli containing important formulations for further trials.

Table 1. Excerpts of ayurvedic formulations containing Kshirakakoli.

\begin{tabular}{|c|c|c|c|}
\hline S. No. & Formulation & Shloka's & References \\
\hline 1. & Jeevaneeya Gana & $\begin{array}{l}\text { तद्यथा - जीवकर्षभकौ मेदा महामेदा } \\
\text { काकोली क्षीरकाकोली मुदपर्णीमाषपण्यो } \\
\text { जीवन्ती मधुकमिति दशेमानि जीवनीयानि भवन्ति }\end{array}$ & Hebbar, 2015a \\
\hline 2. & Shukrala / Kamdev ghrita & $\begin{array}{l}\text { शुक्रलैर्जीवनीयैश्च बृंहणैर्बलवर्धनैः| } \\
\text { क्षीरसज्जननैश्चैव पयः सिद्धं पथथक् पृथक् ॥६॥ } \\
\text { युक्त्तं गोधूमचूर्णेन सघृतक्षौद्र शर्करम्| } \\
\text { पर्यायेण प्रयोक्तव्यमिच्छता शुक्रमक्षयम्||७\| }\end{array}$ & Hebbar, 2015a \\
\hline 3. & $\begin{array}{l}\text { Khuddaka Taila/ khuddaka } \\
\text { padmaka tailam }\end{array}$ & $\begin{array}{l}\text { पद्मकोशीर यष्ट्याह्व रजनी क्राथ साधितम्| } \\
\text { स्यात् पिष्टै: सर्ज मज्ञिष्ठा वीरा काकोलि चन्दनैः||११४| } \\
\text { खुड्डाक पद्मकमिदं तैलं वातास्र दाहनुत्|११५| } \\
\text { इति खुड्डाक पद्मकं तैलम्| }\end{array}$ & Hebbar, 2016a \\
\hline
\end{tabular}




\begin{tabular}{|c|c|c|c|}
\hline 4. & Madhuparnyadi Taila & $\begin{array}{l}\text { मधुकस्य शतं द्राक्षा खर्जूराणि परूषकम्| } \\
\text { मधूकौदनपाक्यौ च प्रस्थं मुआ्जातकस्य च||९६\| } \\
\text { काश्मर्याढकमित्येतच्चतुर्द्रोणे पचेदपाम्| } \\
\text { शेषेऽष्टभागे पूते च तस्मिंस्तैलाढकं पचेत्||९७\| } \\
\text { तथाडSमलक काश्मर्य विदारीक्षु रसैः समैः| } \\
\text { चतुर्द्रोणेन पयसा कल्कं दत्त्ता पलोन्मितम्||९८\| } \\
\text { कदम्बामलकाक्षोट पद्म बीज कशेरुकम्| }\end{array}$ & Hebbar, 2016a \\
\hline 5. & Mahasneham ghrita & $\begin{array}{l}\text { जीवकर्षभकौ मेदामृष्यप्रोक्तां शतावरीम्| } \\
\text { मधुकं मधुपर्णीं च काकोलीद्वयमेव च||७२॥ } \\
\text { मुद्र माषाख्यपर्णिन्यौ दशमूलं पुनर्नवाम्| } \\
\text { बलामृता विदारीश्च साश्वगन्धाश्मभेदकाः |७३॥ } \\
\text { एषां कषाय कल्काभ्यां सर्पिस्तैलं च साधयेत्| } \\
\text { लाभतश्च वसा मज्ज धान्व प्रातुद वैष्किरम्||७४॥ } \\
\text { चतुर्गुणेन पयसा तत् सिद्धं वात शोणितम्| }\end{array}$ & Hebbar, 2016a \\
\hline 6. & $\begin{array}{l}\text { Paste prepared of drugs } \\
\text { belonging to Jivaniya group, } \\
\text { cow-milk and muscle fat/ } \\
\text { Dhanwantharam Thailam }\end{array}$ & $\begin{array}{l}\text { समूलाग्रच्छदैरण्डक्राथे द्वि प्रास्थिकं पृथक्| } \\
\text { घृतं तैलं वसा मज्जा चानूप मृग पक्षिणाम्||१४१॥ } \\
\text { कल्कार्थे जीवनीयानि गव्यं क्षीरमथाजकम्| } \\
\text { हरिद्रोत्पल कुष्ठला शताह्वाश्वहनच्छदान् ॥१४२॥ } \\
\text { बिल्व मात्रान् पृथक् पुष्पं काकुभं चापि साधयेत्| } \\
\text { मधूच्छिष्ट पलान्यष्टौ दद्याच्छीतेऽवतारिते ||२४३\| }\end{array}$ & Hebbar, 2016a \\
\hline 7. & Sneha Parisheka & $\begin{array}{l}\text { स्नेहै र्मधुर सिद्धैर्वा चतुर्भि: परिषेचयेत्| } \\
\text { स्तम्भाक्षेपक शूलार्तं कोष्पैद्दहि तु शीतलैः॥२२५\| }\end{array}$ & Hebbar, 2016a \\
\hline 8. & Parushaka Ghrita & $\begin{array}{l}\text { त्रायन्तिका तामलकी द्विकाकोली शतावरी| } \\
\text { कशेरुका कषायेण कल्केरेभिः पचेद्धृतम्|॥८\| } \\
\text { दत्त्वा परूषका द्राक्षा काश्मर्येक्षुरसान् समान्| } \\
\text { पृथग्विदार्याः स्वरसं तथा क्षीरं चतुर्गुणम्|५९\| } \\
\text { एतत् प्रायोगिकं सर्पिः पारूषकमिति स्मृतम्| } \\
\text { वातरक्ते क्षते क्षीणे वीसर्पे पैत्तिके ज्वरे|६०॥ } \\
\text { इति पारूषकं घृतम्| }\end{array}$ & Hebbar, 2016a \\
\hline 9. & Bala Ghrita/Taila & $\begin{array}{l}\text { बलामतिबलां मेदामात्मगुप्तां शतावरीम्| } \\
\text { काकोलीं क्षीरकाकोलीं रास्नामृद्धिं च पेषयेत्||५६॥ } \\
\text { घृतं चतुर्गुण क्षीरं तैः सिद्धं वातरक्तनुत्| } \\
\text { हृत्पाण्डुरोग वीसर्प कामला ज्वर नाशनम्|५७॥ }\end{array}$ & Hebbar, 2016a \\
\hline 10. & $\begin{array}{l}\text { Sukumara taila/ } \\
\text { Sukumara kashaya }\end{array}$ & 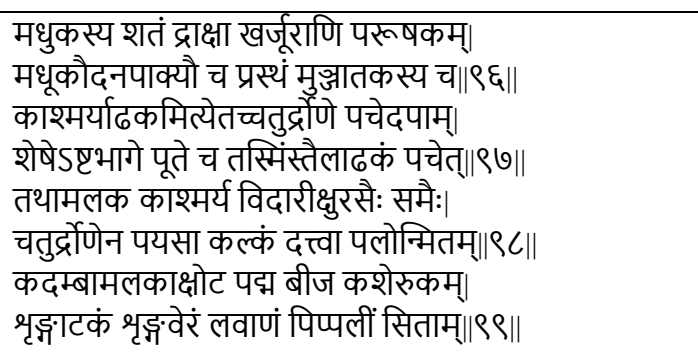 & Hebbar, 2016a \\
\hline 11. & $\begin{array}{l}\text { Snehopaga gana/ (Adjuvants of } \\
\text { Snehana/oleation treatment) }\end{array}$ & $\begin{array}{l}\text { मृद्वीका मधुक मधुपर्णी मेदाविदारी काकोली क्षीरकाकोली } \\
\text { जीवक जीवन्ती शालपर्ण्य इति दशेमानि स्नेहोपगानि भवन्ति }\end{array}$ & Hebbar, 2016a \\
\hline 12. & $\begin{array}{l}\text { Shukrala Shukrajanana/ } \\
\text { Shukra-Shodhana-Janana } \\
\text { Granules }\end{array}$ & $\begin{array}{l}\text { जीवकर्षभक काकोली क्षीरकाकोली } \\
\text { मुदपर्णी माषपर्णी मेदावृद्धरूहा जटिला कुलिड्गा } \\
\text { इति दशेमानि शुक्रजननानि भवन्ति }\end{array}$ & Hebbar, 2016a \\
\hline 13. & Jivaniya Mahakashaya & $\begin{array}{l}\text { तैलप्रस्थं घृतप्रस्थं जीवनीयैः पलोन्मितैः| } \\
\text { क्षीरद्रोणे पचेत् सिद्धमपस्मारविनाशनम्||२८॥ }\end{array}$ & Hebbar, 2016a \\
\hline 14. & $\begin{array}{l}\text { Amruta Prasha Ghruta/ } \\
\text { Amritaprasha Ghrita }\end{array}$ & $\begin{array}{l}\text { जीवकर्षभकौ वीरां जीवन्तीं नागरं शटीम्| } \\
\text { चतस्रः पर्णिनीर्मेदे काकोल्यौ द्वे निदिग्धिके॥३५॥ } \\
\text { पुनर्नवे द्वे मधुकमात्मगुप्तां शतावरीम्| } \\
\text { ऋद्धिं परूषकं भार्गीं मृद्वीकां बृहतीं तथा॥३६॥ } \\
\text { शृड्र्गटकं तामलकीं पयस्यां पिप्पलीं बलाम्| } \\
\text { बदराक्षोट खर्जूर वातामाभिषुकाण्यपि॥३७॥ }\end{array}$ & Hebbar, 2015b \\
\hline
\end{tabular}




\begin{tabular}{|c|c|c|c|}
\hline 15. & $\begin{array}{l}\text { Phalakalyan grita/ Taila Uttara } \\
\text { Basti }\end{array}$ & $\begin{array}{l}\text { कर्णिन्य चरणाशुष्क्योनि प्राक्वरणासु च||१०२॥ } \\
\text { कफवाते च दातव्यं तैलमुत्तर बस्तिना| }\end{array}$ & Hebbar, 2016b \\
\hline 16. & Siva Gutika/Shiva gritham & $\begin{array}{l}\text { मेदां पयस्यां जीवन्तीं विदारीं कण्टकारिकाम्| } \\
\text { श्वदंष्ट्रं क्षीरिकां माषान् गोधूमाज्छालिषष्टिकान्||C\| } \\
\text { पयस्यर्धोदके पक्त्वा कार्षिकानाढकोन्मिते| } \\
\text { विवर्जयेत् पयःशेषं तत् पूतं क्षौद्रसर्पिषा||९\| } \\
\text { युक्तं सशर्करं पीत्वा वृद्धः सप्ततिकोऽपि वा| } \\
\text { विपुलं लभतेऽपत्यं युवेव च स हृष्यति||१०\| }\end{array}$ & Hebbar, 2016b \\
\hline 17. & Vrishya Pooplika & $\begin{array}{l}\text { फलानां जीवनीयानो स्त्रिग्धानां रूचिकारिणाम् । } \\
\text { कुडवश्रूणितानां स्यात् स्वयड्गुमाफलस्य च ॥ १५ ॥ } \\
\text { कुडवश्चैव माषाणां द्वौ च तिलमुद्यो :। } \\
\text { गोघूमशालिचूर्णानां कुडव: कुडवो भवेत् ॥ १६ ॥ }\end{array}$ & Hebbar, 1998 \\
\hline 18. & Upatyakari Shashtikadi Gutika & $\begin{array}{l}\text { आसिक्त क्षीरमापूर्णमशुष्कं शुद्ध षष्टिकम्| } \\
\text { उदूखले समापोथ्य पीडयेत् क्षीरमर्दितम्||३|| } \\
\text { गृहीत्वा तं रसं पूतं गव्येन पयसा सहा| } \\
\text { बीजानामात्मगुप्ताया धान्य माष रसेन च॥४|| }\end{array}$ & Hebbar, 2016c \\
\hline 19. & $\begin{array}{l}\text { Mahamayura Ghrita/ } \\
\text { Mahanarayan Tailm }\end{array}$ & $\begin{array}{l}\text { एतेनैव कषायेण घृत प्रस्थं विपाचयेत्| } \\
\text { चतुर्गुणेन पयसा कल्कैरेभिश्च कार्षिकै:\|१६६॥ } \\
\text { जीवन्ती त्रिफला मेदा मृद्वीकर्धि परूषकैः| } \\
\text { समड़ग चविका भार्गी काश्मरी सुरदारुभिः |१६७\| } \\
\text { आत्मगुप्ता महामेदा ताल खर्जूर मस्तकैः| }\end{array}$ & Hebbar, 2015c \\
\hline 20. & Jivantyadi Ghrita & $\begin{array}{l}\text { एवमेव क्षीर सर्पि जीवनीयोपसाधितम्||६९\| } \\
\text { गर्भदं पित्तलानां च योनीनां स्याद्दिषग्जितम्|७०| }\end{array}$ & Hebbar, 2015c \\
\hline
\end{tabular}

Table 2. Compliance level of therapeutic uses of kshirakakoli containing formulations as per ancient literature with trado-medical practitioners (TMPs) and Pre-clinical/clinical trials/case studies as per modern system of medicine.

\begin{tabular}{|c|c|c|c|c|}
\hline Sr. No. & Formulations & Therapeutic uses as per texts & $\begin{array}{l}\text { Therapeutic uses as } \\
\text { mentioned by trado-medical } \\
\text { practitioners (TMPs) }\end{array}$ & $\begin{array}{l}\text { Pre-clinical/clinical } \\
\text { trials/case studies as per } \\
\text { modern system of medicine }\end{array}$ \\
\hline \multirow[t]{15}{*}{1.} & Amruta Prasha & 1. Cough & Yes & No \\
\hline & Ghruta/ & 2. Hiccup & Yes & No \\
\hline & Amritaprasha & 3. Fever & Yes & No \\
\hline & Ghrita & 4. Asthma & Yes & No \\
\hline & & 5. Burning sensation & Yes & No \\
\hline & & 6. Morbid thirst & Yes & No \\
\hline & & $\begin{array}{l}\text { 7. An ailment characterized by } \\
\text { bleeding from different parts of } \\
\text { the body }\end{array}$ & Yes & No \\
\hline & & 8. Vomiting & Yes & No \\
\hline & & 9. Fainting & Yes & No \\
\hline & & 10. Diseases of heart & No & No \\
\hline & & 11. Female genetial tract & Yes & No \\
\hline & & 12. Urinary tract & No & No \\
\hline & & 13. Procreation of male child & Yes & No \\
\hline & & 14. Joint problems and other & Yes & Yes \\
\hline & & $\begin{array}{l}\text { diseases caused by unbalanced } \\
\text { vata \& pitta doshas. }\end{array}$ & & $\begin{array}{l}\text { Improves the nonspecific } \\
\text { immunity against rheumatoid } \\
\text { arthritis (Lekurwalel et al., } \\
\text { 2010). }\end{array}$ \\
\hline \multirow[t]{3}{*}{2.} & Jeevaneeya Gana & 1. Enlivening & Yes & No \\
\hline & & 2. Anti-aging & Yes & No \\
\hline & & 3. Antioxidant & Yes & No \\
\hline \multirow[t]{6}{*}{3.} & Jivantyadi Ghrita & 1. Cure Female infertility & Yes & No \\
\hline & & 2. Cure Cataract and Glaucoma & Yes & Yes \\
\hline & & & & Effective in treatment and \\
\hline & & & & controlling of Computer \\
\hline & & & & $\begin{array}{l}\text { V1sion Syndrome and helpful } \\
\text { in curing myopia (Vinaik et }\end{array}$ \\
\hline & & & & al., 2013; Shukla et al., 2011). \\
\hline
\end{tabular}




\begin{tabular}{|c|c|c|c|c|}
\hline 4. & $\begin{array}{l}\text { Khuddaka Taila/ } \\
\text { khuddaka } \\
\text { padmaka tailam }\end{array}$ & $\begin{array}{l}\text { 1. Gout } \\
\text { 2. Burning sensation }\end{array}$ & $\begin{array}{l}\text { Yes } \\
\text { Yes }\end{array}$ & $\begin{array}{l}\text { No } \\
\text { No }\end{array}$ \\
\hline 5. & $\begin{array}{l}\text { Madhuparnyadi } \\
\text { Taila }\end{array}$ & $\begin{array}{l}\text { 1. Cures Vatarakta } \\
\text { 2. Pain in limbs } \\
\text { 3. Affliction of the whole body } \\
\text { 4. It also promotes strength and } \\
\text { complexion. }\end{array}$ & $\begin{array}{l}\text { Yes } \\
\text { Yes } \\
\text { Yes } \\
\text { Yes }\end{array}$ & $\begin{array}{l}\text { No } \\
\text { No } \\
\text { No } \\
\text { No }\end{array}$ \\
\hline 6. & $\begin{array}{l}\text { Mahamayura } \\
\text { Ghrita/ } \\
\text { Mahanarayan } \\
\text { Tailm }\end{array}$ & $\begin{array}{l}\text { 1. Neuro-muscular disorders } \\
\text { 2. Cough } \\
\text { 3. Asthma } \\
\text { 4. Inflammation } \\
\text { 5. Diseases of the female genital } \\
\text { tract } \\
\text { 6. Menstrual disorders }\end{array}$ & $\begin{array}{l}\text { Yes } \\
\text { Yes } \\
\text { Yes } \\
\text { Yes } \\
\text { Yes }\end{array}$ & $\begin{array}{l}\text { Yes } \\
\text { No } \\
\text { No } \\
\text { Yes } \\
\text { Yes } \\
\text { Used to treat inflammation, } \\
\text { pain and arthritis, Useful in } \\
\text { treatment of chronic back } \\
\text { pain, Useful in treatment of } \\
\text { female infertility, provide } \\
\text { strength to the local soft } \\
\text { tissues, thus play significant } \\
\text { role in the management of } \\
\text { cervical spondylosis or } \\
\text { osteoarthritis of cervical spine } \\
\text { (Pawar et al., 2011; Panda } \\
\text { and Debnath, 2011; Kaushik } \\
\text { et al., 2017; Kunjibettu et al., } \\
\text { 2017). }\end{array}$ \\
\hline 7. & $\begin{array}{l}\text { Mahasneham } \\
\text { ghrita }\end{array}$ & $\begin{array}{l}\text { 1. Gout } \\
\text { 2. Serious diseases caused by the } \\
\text { aggravated Vata } \\
\text { 3. Arthritis }\end{array}$ & $\begin{array}{l}\text { Yes } \\
\text { Yes }\end{array}$ & $\begin{array}{l}\text { No } \\
\text { No }\end{array}$ \\
\hline 8. & $\begin{array}{l}\text { Paste prepared of } \\
\text { drugs belonging } \\
\text { to Jivaniya } \\
\text { group, cow-milk } \\
\text { and muscle fat/ } \\
\text { Dhanwantharam } \\
\text { Thailam }\end{array}$ & $\begin{array}{ll}\text { 1. } & \text { Cures pain } \\
\text { 2. } & \text { Gout } \\
\text { 3. } & \text { Kyphosis (Spinal disorder) }\end{array}$ & $\begin{array}{l}\text { No } \\
\text { Yes } \\
\text { Yes }\end{array}$ & $\begin{array}{l}\text { No } \\
\text { No } \\
\text { No }\end{array}$ \\
\hline 9. & $\begin{array}{l}\text { Phalakalyan } \\
\text { grita/ Taila } \\
\text { Uttara Basti }\end{array}$ & $\begin{array}{l}\text { 1. Uterine Tonic } \\
\text { 2. Gynecological disorders }\end{array}$ & $\begin{array}{l}\text { Yes } \\
\text { Yes }\end{array}$ & $\begin{array}{l}\text { No } \\
\text { Yes } \\
\text { Useful in treatment of female } \\
\text { infertility (Kunjibettu et al., } \\
\text { 2017). }\end{array}$ \\
\hline 10. & $\begin{array}{l}\text { Shukrala/ } \\
\text { Kamdev Ghrita }\end{array}$ & $\begin{array}{l}\text { 1. Improves semen and sperm } \\
\text { production }\end{array}$ & Yes & No \\
\hline 11. & $\begin{array}{l}\text { Siva } \\
\text { Gutika/Shiva } \\
\text { gritham }\end{array}$ & $\begin{array}{l}\text { 1. Diabetes mellitus } \\
\text { 2. Bronchitis } \\
\text { 3. Anemia } \\
\text { 4. Cardiac diseases } \\
\text { 5. Epilepsy } \\
\text { 6. Psychotic diseases } \\
\text { 7. Skin diseases } \\
\text { 8. Infertility in women/men } \\
\text { 9. Liver diseases }\end{array}$ & $\begin{array}{l}\text { No } \\
\text { No } \\
\text { Yes } \\
\text { Yes } \\
\text { Yes } \\
\text { Yes } \\
\text { No } \\
\text { Yes } \\
\text { Yes }\end{array}$ & $\begin{array}{l}\text { No } \\
\text { No } \\
\text { No } \\
\text { No } \\
\text { No } \\
\text { No } \\
\text { No } \\
\text { No } \\
\text { Yes } \\
\text { Used in treatment of chronic } \\
\text { liver and highly effective in } \\
\text { treatment of HIV/AIDS and } \\
\text { Acute Deep Vein Thrombosis } \\
\text { (Wasedar et al., 2017; Rathod } \\
\text { et al., 2013; Pusuluri et al., } \\
\text { 2017). }\end{array}$ \\
\hline 12. & Sneha Parisheka & $\begin{array}{l}\text { 1. Stiffness } \\
\text { 2. Convulsion } \\
\text { 3. Gout } \\
\text { 4. Burning sensation }\end{array}$ & $\begin{array}{l}\text { Yes } \\
\text { Yes } \\
\text { Yes } \\
\text { Yes }\end{array}$ & $\begin{array}{l}\text { No } \\
\text { No } \\
\text { No } \\
\text { No }\end{array}$ \\
\hline
\end{tabular}




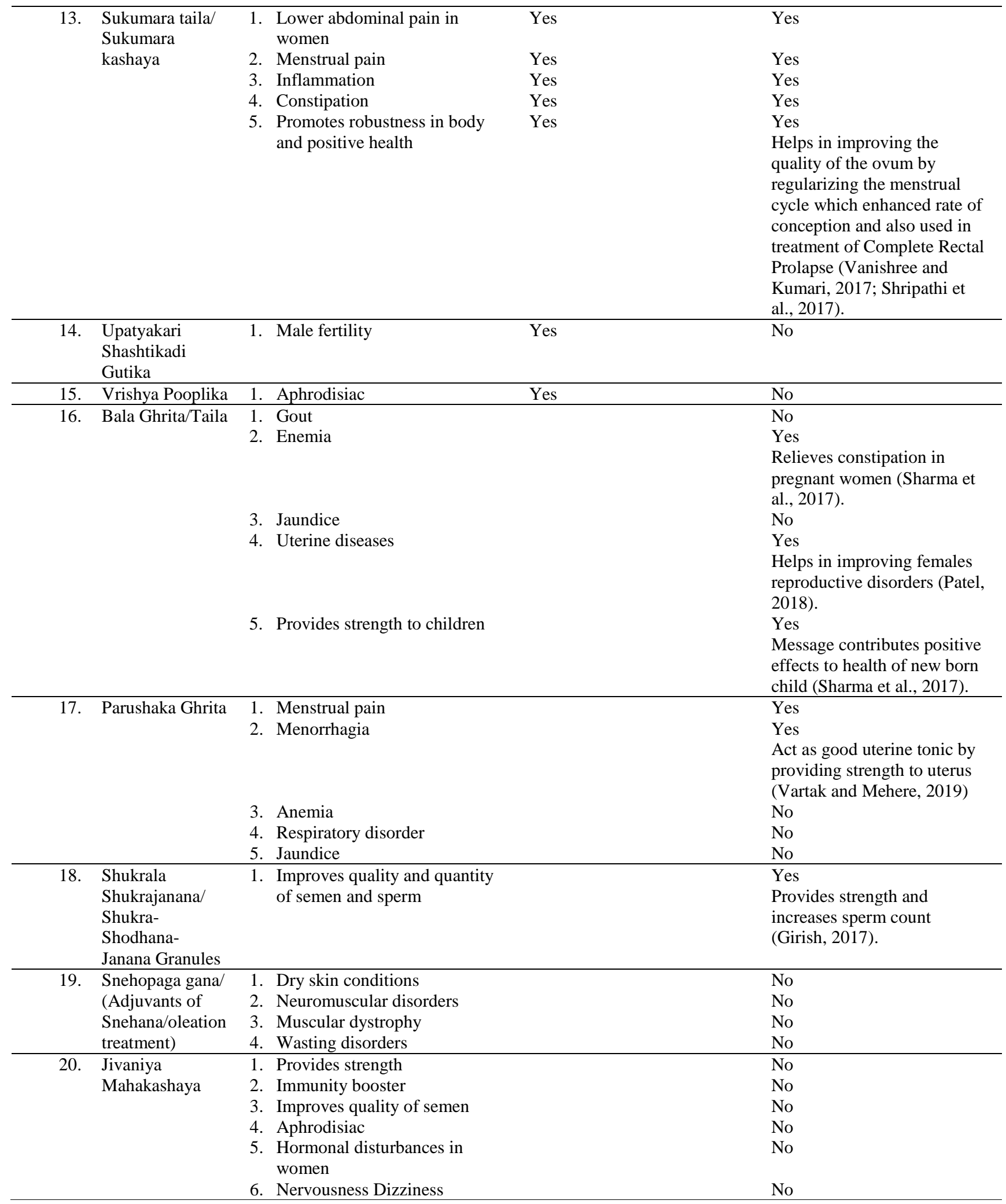

\section{CONCLUSION}

The analysis of literature reveals that Fritillaria roylei is a wonder plant used by Saints/Rishies since ages however due to a number of reasons; plant has been ignored for its therapeutic uses. This survey clearly indicates that there is a need of deciphering the textual references given in regional languages and use them for new drug development process. As clearly indicated in textual as well as in survey, most of the Kshirakakoli 
containing formulations have been recommended for number of disorders in men and women. However, there is lack of such patent formulations as well systemized clinical trials that could prove to be useful in highlighting the real therapeutic potentials of Kshirakakoli containing formulations. Hence this survey provides a template for scientists for further screening and research on these formulations that are useful in plethora of disorders.

\section{Financial support: None}

Acknowledgment: The authors are thankful to the tradomedical practitioners (TMPs) for sharing their precious traditional knowledge.

Conflict of interest: The authors declares that there are no conflicts of interest concerning the publication of this article.

\section{REFERENCES}

Anesini, C., Perez, C. (1993). Screening of plants used in Argentine folk medicine for antimicrobial activity. Journal of ethnopharmacology, 39(2) 119-128. https://doi.org/ 10.1016/0378-8741 (93) 90027-3.

Chatterjee, A., Dhara, K.P., Pascard, C., Prange, T. (1976). Kashmirine, a new steroidal alkaloid from Fritillaria roylei, Hook (Liliaceae). Tetrahedron Lett., 33 2903-2904.

Chi, Y.F., Kao, Y.S., Chang, K.J. (1940). The Alkaloids of Fritilaria Roylei. II. Isolation of Peiminine1. Journal of the American Chemical Society, 62(11) 2896-2897. DOI: https://doi.org/10.1021/ja01868a002

Chou, T.Q. (1947). Some minor alkaloids of pei-mu, Fritillaria roylei. J. Am. Pharma. Assoc., 36(7) 215-217. DOI: https://doi.org/10.1002/jps.3030360707.

Dhyani, A., Nautiyal, B.P., Nautiyal, M.C. (2010). Importance of Astavarga plants in traditional systems of medicine in Garhwal, Indian Himalaya. Int. J. Biodiv. Sc., Ecosystem Services \& Management, 6(1-2)13-19. DOI: https://doi.org/10.1080/ 21513732.2010 .521490$.

Girish, K.J. (2017). Clinical Study on Effectiveness of ShukraShodhana-Janana Granules and Erandmula Kvatha in Patients of Oligospermia. Journal of Ayurveda Physicians \& Surgeons, 4(3) 35-38. ISSN 2394- 6350

Hebbar, J.V. (2016b). Charaka Samhita Chikitsa Sthana; Vatavyadhi Chikitsa Adhyaya: Chapter 28, http://www.easyayurveda.com/2016/01/03/charakavatavyadhi-chikitsa-28/2016b. [Date last accessed 03 March 2018].

Hebbar, J.V. (1998). Charak Samhita, Chaukhambha Orientalia Varanasi, Volume II, Section 6 Chikitsasthanam, Chapter II, Qtr 3 (2.3), Shloka 15-17 45.

Hebbar, J.V. (2015a). Charak Samhita Sutrasthana; Mashaparna Bhrutiya Adhyaya Charaka Chikitsa 2.3, http://www.easyayurveda.com/2015/07/21/charaka-chikitsa2.3-chapter-mashaparna-bhrutiya/2015a. [Date last accessed 03 March 2018].
Hebbar, J.V. (2015b). Charaka Samhita Chikitsa; Charaka Kshatasheena Chikitsa: Chapter 11 , http://www.easyayurveda.com/2015/09/23/charakakshatasheena-chikitsa/. 2015b. [Date last accessed 03 March 2018].

Hebbar, J.V. (2015c). Charaka Chikitsa; Trimarmeeya Chikitsa: Chapter 26, http://www. easyayurveda.com /2015/12/22/ trimarmeeya-chikitsa-charaka-26/2015c. [Date last accessed 03 March 2018].

Hebbar, J.V. (2016a). Charaka Samhita Chikitsa Sthana; Charaka Samhita Vatarakta Chikitsa: Chapter 29, http://www.easyayurveda.com /2016/01/06/ charakavatarakta-chikitsa-29/2016a. [Date last accessed 03 March 2018].

Hebbar, J.V. (2016c). Charaka Samhita Sutrasthana Asikta Kshiriya Vajikarana Pada: Charak Chikitsa 2.2, http://www.easyayurveda.com/2016/02/02/charaka-chikitsasthana-30th-chapter-yoni-vyapat/2016c. [Date last accessed 03 March 2018].

Jiang, Y., Li, P., L,i H.J., Yu, H. (2006). New steroidal alkaloids from the bulbs of Fritillaria puqiensis. Steroids, 71(9) 843848. DOI: https://doi.org/ 10.1016/j. steroids. 2006.05.016

Kaur, G., Gupta, V., Kumar, S., Singhal, R.G., Maithani, M., Bansal, P. (2019). Compliance level of textual therapeutic usage of kakoli-containing formulations with ethnomedicinal survey and modern system of medicine. Imam Journal of Applied Sciences, 4(2) 62-68. DOI: https://doi.org/ 10.4103/ijas.ijas_1_19.

Kaushik, R., Sharma, P., Prakash, O. (2017). Management of Cervical Spondylosis through Ayurveda: A Case Study. Int. J. Res. Ayu. Pharm. 8(2) 179-181. DOI: https://doi.org//10.7897/2277-4343.082109

Kshiti, S. (2012). Introduction of Ayurveda and Human Potential: The First Life Science. Journal of Phytopharmacology, 1(2) 1-5. ISSN: 2320-480X

Kunjibettu, S., Chaudhari, K., Dei, L. (2017). Role of Uttarabasti in the Management of Female Infertility-Evidence Based Critical Review. J. Ayu. \& Holistic Med., 5(1) 1-12. ISSN2321-1563

Lekurwalel, P.S., Pandey, K., Yadaiah, P. (2010). Management of Amavata with 'Amrita Ghrita': A clinical study. AYU, 31(4) 430-435. DOI: https://doi.org/ 10.4103/0974-8520. 82033.

Panda, A.K., Debnath, S.K. (2011). Effectiveness of Kativasthi and excersice in chronic low back pain: A Randomized control study. Int. J. Resh. Ayu. Pharm. 2(2) 338-342.

Patel, J. (2018). Clinical Efficacy of Bala Taila Uttarbasti the Management of Urethral Stricture: Case Report. Int. J. Curr. Resh. 10(8) 73068-73071. DOI: https://doi.org /10.24941/ ijcr.32201.08.2018.

Pawar, S.D., Gaidhani, S.N., Anandan, T. (2011). Evaluation of Anti Inflammatory, Analgesic and Anti-Arthritic Activity of Mahanarayana Tailam in Laboratory Animals. Adv. Pharmacol. Toxicol., 12(3) 33-42. ISSN - 0973-2381

Pusuluri, Y.V.S.M.K., Wasedar, V.S., Dani, H. (2017). Effect of punarnavadi Mandoor and Shiva Gutika in Acute Deep Vein Thrombosis- A Case Report. J. Ayu. Integ. Med. Sci., 2(4) 296-298. DOI: https://doi.org/10.21760/jaims.v2i4.9371.

Rathod, M., Mutha, R., Murgesh, S., Sharma, A., Acharya, G.S., Sharma, A.K. (2013). Clinical Study to Evaluate the Therapeutic Effect of Shivagutika in Patients with H.I.V. Int. Ayu. Med. J. 1(3) 1-7. ISSN:2320- 5091

Sagar, P.K. (2014). Adulteration and substitution in endangered, ASU herbal medicinal plants of India, their legal status, 
scientific screening of active phytochemical constituents. Int. J. Pharm. Sci. Res., 5(9) 4023-4039. DOI: https://doi.org/ 10.13040/IJPSR.0975-8232.5 (9).4023-4039.

Saha, D., Ved, D., Ravikumar, K., Haridasan, K., Dhyani, A. (2015). Lilium polyphyllum. The IUCN Red List of Threatened Species, 2015. Available at: www.iucnredlist.org. [Date last accessed 25, Nov, 2019].

Sharma, B.D., Balkrishna, A.V. (2005). Vitality strengthening Astavarga plants (Jeevaniya \& Vayasthapan Paudhe), Divya Publishers, Uttaranchal (India).

Sharma, P., Nariyal, V., Sharma, S., Sharma, A. (2017). Reproductive and Child Health Care through Bala Taila: A Review on Ayurveda Formulation. Int. J. Res. Ayurveda Pharm. 8(3) 1-4. DOI: https://doi.org/10.7897/22774343.083132 .

Shripathi, A.H., Malagi, K.J., Hebbar, K.R. (2017). Evaluating the Importance of Bahya and Abhyantara Prayoga of Mushika Taila in Complete Rectal Prolapse: A Case Study. Int. J. Ayu. Pharm. Chem. 7(2) 120-128. ISSN 2350-0204.

Shukla, V. J., Poonam Manjusha, R., Vaghela, D. B. (2011). A clinical study on the role of Akshi Tarpana with Jeevantyadi Ghrita in Timira (Myopia). AYU, 32(4) 540-545. DOI: https://doi.org/10.4103/0974-8520.96130.
Tewari, N.N. (1991). Some crude drugs: source, substitute and adulterant with special reference to KTM crude drug market. Sachitra Ayurved, 44(4) 284-290.

Vanishree, S.K., Kumari, C.A. (2017). Treating Post Salphyngectomy Infertility with Ayurveda- A Case Study. World J. of Pharmacy and Pharmaceutical Sci., 6(12) 14451453. DOI: https://doi.org/10.20959/wjpps201712-10584.

Vartak, R.V., Mehere, S. (2019). Effect of ashoka ghrita in asrigdhar (menorrhagia): A case study. Ayur log: National $J$. Research in Ayurved Science, 3(2) 1-5. ISSN: 2320-7379

Vinaik, A., Kataria, A., Singhal, H.K., Kumar, K. (2013). A Clinical Study on Jeevaniya Ghrita and Drishti Prasadaka Yoga (Formulated Compound) in the Management of Computer Vision Syndrome (CVS) w.s.r Shushkakshipaka. Int. Ayurvedic Medical Journal, 1(6) 154-162. DOI: https://doi.org/10.4103/0974-8520.108831

Wasedar, V.S., Kapoor, T., Rao, N. (2017). Chronic Liver Disease Management in Ayurveda W.S.R. to Garavisha (Artificial Poison) - A Case Report. Int. Ayu. Med. J. 1(3) 364-368. ISSN: 2320- 5091

Wu, Y.H. (1944). The Constituents of Fritillaria roylei. J. Am. Chem. Soc., 66(10) 1778-1780. DOI: https://doi.org/10.1021/ja01238a048. 